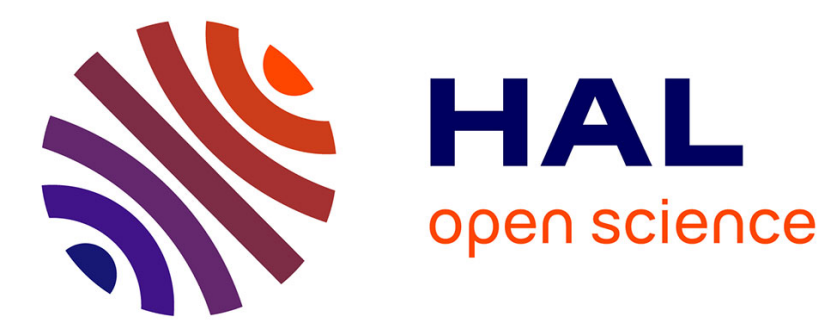

\title{
High frequency trading in a Markov renewal model
} Pietro Fodra, Huyen Pham

\section{To cite this version:}

Pietro Fodra, Huyen Pham. High frequency trading in a Markov renewal model. 2013. hal-00867113

\section{HAL Id: hal-00867113 \\ https://hal.science/hal-00867113}

Preprint submitted on 27 Sep 2013

HAL is a multi-disciplinary open access archive for the deposit and dissemination of scientific research documents, whether they are published or not. The documents may come from teaching and research institutions in France or abroad, or from public or private research centers.
L'archive ouverte pluridisciplinaire HAL, est destinée au dépôt et à la diffusion de documents scientifiques de niveau recherche, publiés ou non, émanant des établissements d'enseignement et de recherche français ou étrangers, des laboratoires publics ou privés. 


\title{
High frequency trading in a Markov renewal model
}

\author{
Pietro FODRA \\ Laboratoire de Probabilités et \\ Modèles Aléatoires \\ CNRS, UMR 7599 \\ Université Paris 7 Diderot \\ and EXQIM \\ pietro.fodra91@gmail.com
}

\author{
Huyên PHAM \\ Laboratoire de Probabilités et \\ Modèles Aléatoires \\ CNRS, UMR 7599 \\ Université Paris 7 Diderot \\ pham@math.univ-paris-diderot.fr \\ CREST-ENSAE \\ and JVN Institute, Ho Chi Minh City
}

September 27, 2013

\begin{abstract}
We study an optimal high frequency trading problem within a market microstructure model aiming at a good compromise between accuracy and tractability. The stock price is modeled by a Markov Renewal Process (MRP) as described in [12], while market orders arrive in the limit order book via a point process correlated with the stock price, and taking into account the adverse selection risk. We apply stochastic control methods in this semi-Markov framework, and show how to reduce remarkably the complexity of the associated Hamilton-Jacobi-Bellman equation by suitable change of variables that exploits the specific symmetry of the problem. We then handle numerically the remaining part of the HJB equation, simplified into an integro-ordinary differential equation, by a bidimensional Euler scheme. Statistical procedures and numerical tests for computing the optimal limit order strategies illustrate our results.
\end{abstract}

Keywords: High frequency trading, Markov renewal process, Marked Cox process, adverse selection, integro-ordinary differential equation. 


\section{Introduction}

The literature on high frequency in finance can be basically divided into two main streams:

1. Models of intra-day asset price: this literature is devoted to the description of a tick-by-tick asset price in a limit order book with essentially two points of view: (i) the latent process approach starts from a macroscopic latent process, typically a diffusion, which is contaminated by a noise reflecting the microstructure effects at high frequency data: discreteness of prices valued in a tick grid, irregular spacing of price jump times (known as volatility clustering), mean-reversion of price variations. Some papers in this direction are [13], [2], and [19]. (ii) The micro-macro approach models directly the observed price by means of point processes, see e.g. [6], [10], [1] or [17]. These papers consider sophisticated models, and are mainly intended to reproduce microstructure stylized facts, as signature plot, Epps effect, volatility clustering and short mean-reversion, and often for purpose of volatility estimation, while trading applications are not studied.

2. High frequency trading problems: another important literature stream focus on trading problems in limit order book: stock liquidation and execution problems ([4], [3], [7], $[16]$, etc ...), or market making problems ([5], [9], [15], [14], [11], [8], etc ...). These papers use stochastic control methods for optimal trading strategies, and are mostly based on classical models for asset price, typically arithmetic or geometric Brownian motion, while the market order flow is usually modeled by a Poisson process, hence independent of the continuous price process.

The goal of this paper is to make a bridge between these two streams of literature, by constructing a simple model for asset price in a limit order book, intended to be both:

- realistic by capturing main stylized facts of microstructure, and easy to estimate and simulate,

- tractable (simple to analyze and implement) for dynamic optimization in high frequency trading.

We shall rely on our previous work [12], where it is shown how a particular class of point processes, the Markov Renewal ones, can be extremely flexible and pertinent to model the stock price at high frequency by reproducing the above mentioned stylized facts, and how it can be easily estimated and simulated. In a second step, we introduce a suitable modeling of market order flow, which takes into account the existing dependence with the stock price dynamics. This is achieved by means of a marked Cox process subordinated to the stock price dynamics, which allows us to consider the adverse selection risk, in the sense that a posted limit order is more likely to be executed if its execution is unfavorable for the market maker. In this context, we study the market making problem of an agent submitting optimally limit orders at best bid and best ask prices. We apply stochastic control methods in this semi-Markov framework, and show how to reduce the complexity of the associated Hamilton-Jacobi-Bellman equation by suitable change of variables that 
exploits the specific symmetry of the problem. The remaining part of the HJB equation is reduced to an integro ordinary differential equation (IODE) involving as state variables: current time, elapsed time since last jump of the stock price, and inventory. We provide a convergent numerical scheme for solving this IODE, and illustrate our results with numerical tests displaying the value function and optimal policy shape for various parameters of the model.

The structure of the paper is organized as follows. We briefly review in Section 2 the asset price model introduced in [12]. In Section 3, we describe the market order flow modeling. We introduce a marked Cox process subordinated to the stock price dynamics in order to reproduce the adverse selection risk and the intensity correlation between the two processes, and then give a parametric estimation procedure. Section 4 formulates the optimal market making problem, and show some estimates bounds on the value function. Section 5 is devoted to the resolution of the market making problem: we provide an analytic characterization of the solution by dynamic programming methods and suitable double change of variables. Finally, some numerical tests illustrate our results.

\section{Stock price in the limit order book}

We consider in this section a model for the mid-price of an asset, i.e the mean between the best-bid price and best-ask price, in a limit order book with a constant bid-ask spread equal to the tick size $2 \delta>0$. For simplicity, we assume that the asset price jumps by only one tick. The mid-price $\left(P_{t}\right)_{t \geq 0}$ is then defined by the càd-làg piecewise constant process:

$$
P_{t}=P_{0}+2 \delta \sum_{n=1}^{N_{t}} J_{n}
$$

where $P_{0}$ is the opening mid-price, $N_{t}$ is the point process associated to the tick times $\left(T_{n}\right)_{n}$ (i.e. the price jump times):

$$
N_{t}=\inf \left\{n: \sum_{k=1}^{n} T_{k} \leq t\right\},
$$

and $\left(J_{n}\right)_{n}$ is the marks sequence valued in $\{+1,-1\}$ indicating whether the price jumped upwards $\left(J_{n}=+1\right)$ or downwards $\left(J_{n}=-1\right)$ at time $T_{n}$, called price direction.

We use a Markov renewal approach as in [12] for modeling the marked point process $\left(T_{n}, J_{n}\right)_{n}$. The price direction is modeled by a Markov chain as:

$$
J_{n}=J_{n-1} B_{n},
$$

where $\left(B_{n}\right)_{n}$ is an i.i.d sequence, independent of $\left(J_{n}\right)$, and distributed according to a Bernoulli law on $\{+1,-1\}$ with parameter $(1+\alpha) / 2, \alpha \in[-1,1)$. In other words, the probability of two consecutive jumps in the same (resp. opposite) direction: $\mathbb{P}\left[J_{n} J_{n-1}= \pm 1\right]$ is equal to $(1 \pm \alpha) / 2$. Moreover, under the stationary measure $\pi=(1 / 2,1 / 2)$ for the Markov chain $\left(J_{n}\right)$, the correlation between two consecutive price directions $J_{n-1}$ and $J_{n}$ is equal

to $\alpha$. When $\alpha=0$, this means that the jumps of the stock price are independent. When $\alpha$ 
$>0$, the price is short-term trended, while for $\alpha<0$, the stock price exhibits a short-term mean-reversion, which is a well-known stylized fact about high-frequency data, also called microstructure noise, and usually met on real data (futures on index, debt and currencies). Next, we model the counting process $\left(N_{t}\right)$. Denoting by $S_{n}=T_{n}-T_{n-1}$ the inter-arrival times of the price jump times, we assume that conditionally on the sign $J_{n} J_{n+1}= \pm 1$ of two consecutive price directions, $\left(S_{n}\right)_{n}$ is an independent sequence with distribution:

$$
F_{ \pm}(s)=\mathbb{P}\left[S_{n+1} \leq s \mid J_{n} J_{n+1}= \pm 1\right]
$$

and density $f_{ \pm}(s)$. We easily see that $\left(S_{n}\right)$ is also unconditionally i.i.d. with distribution given by:

$$
F=\frac{1+\alpha}{2} F_{+}+\frac{1-\alpha}{2} F_{-} .
$$

In other words, $N$ is a renewal process with inter-arrival times distribution $F$.

Let us define the pure jump process valued in $\{1,-1\}$ :

$$
I_{t}=J_{N_{t}}
$$

which gives at time $t$ the direction of the last jump of the stock price. Then $I$ is a semiMarkov process in the sense that the pair $\left(I_{t}, S_{t}\right)$ is a Markov process, where

$$
S_{t}=t-\sup \left\{T_{n}: T_{n} \leq t\right\},
$$

is the time spent from the last price jump. Moreover, the price process $\left(P_{t}\right)$ is embedded into a Markov system with three observable state variables: $\left(P_{t}, I_{t}, S_{t}\right)$ is a Markov process with infinitesimal generator:

$$
\begin{aligned}
\mathcal{L} \varphi(p, i, s)= & \frac{\partial \varphi}{\partial s}+h_{+}(s)[\varphi(p+2 \delta i, i, 0)-\varphi(p, i, s)] \\
& +h_{-}(s)[\varphi(p-2 \delta i,-i, 0)-\varphi(p, i, s)]
\end{aligned}
$$

where

$$
\begin{aligned}
h_{ \pm}(s) & :=\lim _{\Delta s \rightarrow 0^{+}} \frac{1}{\Delta s} \mathbb{P}\left[s \leq S_{n+1} \leq s+\Delta s, J_{n+1}= \pm J_{n} \mid S_{n} \geq s, J_{n}\right] \\
& =\frac{1 \pm \alpha}{2} \frac{f_{ \pm}(s)}{1-F(s)} .
\end{aligned}
$$

represents the intensity function of price jump in the same (resp. opposite) direction.

Remark 2.1. From (2.3), we observe that the elapsed time process $\left(S_{t}\right)_{t}$ is an homogeneous Markov process with infinitesimal generator:

$$
\mathcal{L} \varphi(s)=\frac{\partial \varphi}{\partial s}+\sigma^{2}(s)[\varphi(0)-\varphi(s)]
$$

where

$$
\sigma^{2}:=h_{+}+h_{-}
$$

represents the intensity function of price jump whatever the direction. Moreover, $\left(N_{t}, S_{t}\right)$ is a Markov process, and the conditional intensity of the point process $\left(N_{t}\right)$ is equal to $\sigma^{2}\left(S_{t}\right)$ 
Let us consider the conditional renewal function:

$$
\nu(t, s)=\mathbb{E}\left[N_{t} \mid S_{0}=s\right], \quad t, s \geq 0,
$$

and by misuse of notation, we set: $\nu(t)=\nu(t, 0)$ the renewal function:

$$
\nu(t)=\mathbb{E}\left[N_{t}\right], \quad t \geq 0
$$

which is finite on $\mathbb{R}_{+}$since $N$ is a renewal process. Notice that since $\sigma^{2}\left(S_{t}\right)$ is the conditional intensity of the renewal process $N_{t}$, the conditional renewal function is also equal to:

$$
\nu(t, s)=\mathbb{E}\left[\int_{0}^{t} \sigma^{2}\left(S_{u}\right) d u \mid S_{0}=s\right] .
$$

The following result shows the finiteness of the conditional renewal function.

Lemma 2.1. We have

$$
\nu(t, s) \leq \nu(t)+1<\infty, \quad \forall t, s \geq 0
$$

Proof. Let us introduce $\tau_{1}$ the first jump of $N$ after time 0 . Then, by the Markov property of $(N, S)$, we have:

$$
\nu(t, s)=\mathbb{E}\left[N_{t}-N_{\tau_{1}} \mid S_{\tau_{1}}=0\right]+\mathbb{E}\left[N_{\tau_{1}} \mid S_{0}=s\right] \leq \nu(t, 0)+1,
$$

which shows the required result.

We refer to [12] for the statistical estimation of the trendiness parameter $\alpha$, the distribution $F_{ \pm}$of the renewal times, and the jump intensities $h_{ \pm}$. In the sequel, market data are taken from tick-by-tick observation of the 3-month future EUROSTOXX50, on February 2011, from 09:00:000 to 17:00:00.000 (CET). Figure 1 plots the form of $h_{ \pm}$as a function of the renewal quantile, i.e. the quantile in the distribution $F$ given in (2.2): it shows that the reverting intensity $h_{-}$is dominant w.r.t. to the trending intensity $h_{+}$for small renewals, while this discrepancy tends to disappear for higher quantiles $(>0.50)$, and we even observe that $h_{+}$dominates $h_{-}$for large renewal quantiles close to 1 . This can be interpreted by saying that when the price is stable, i.e. its quotation has been constant for a long time, the microstructural mean reversion disappears, and the price looks like a Bernoulli random walk. 


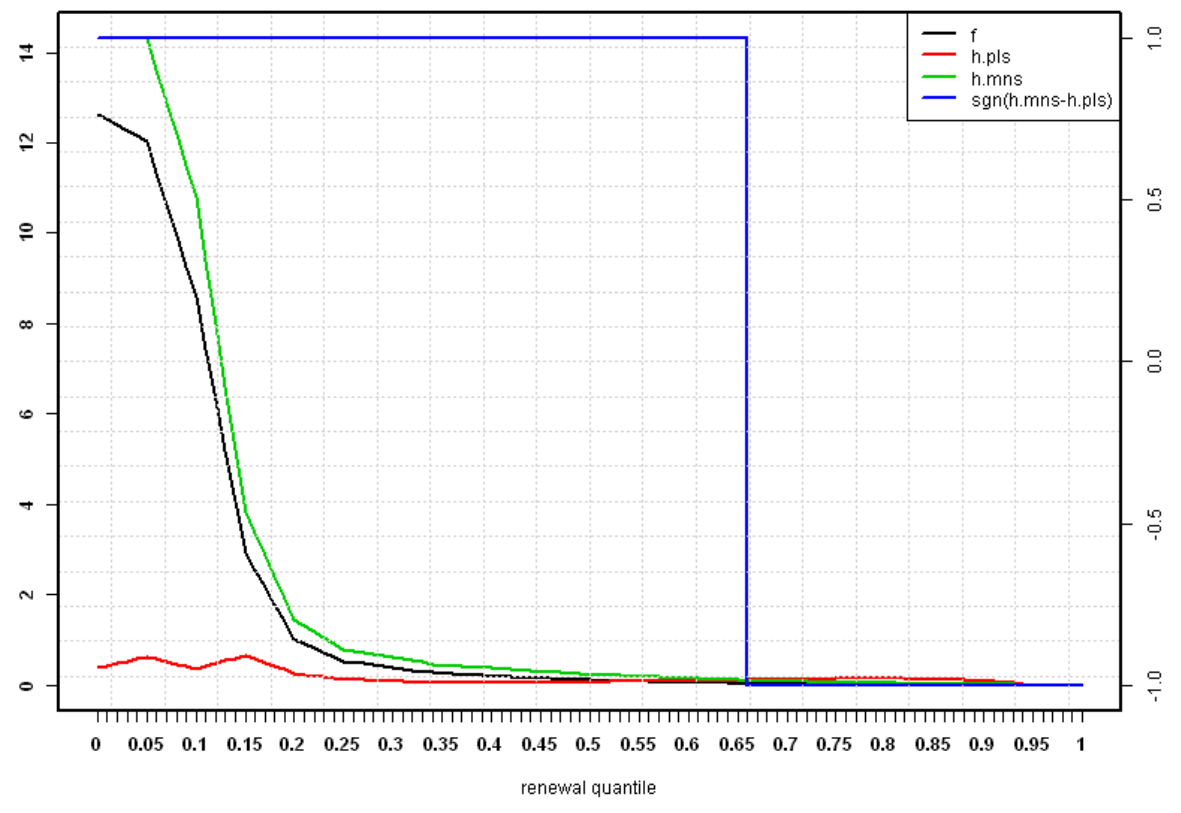

Figure 1: Non parametric estimation of $h_{ \pm}$as a function of the renewal quantile $(\alpha=-0.75)$

\section{Market order flow modeling}

We model the market order flow by a marked point process $\left(\theta_{k}, Z_{k}\right)_{k}$ where the increasing sequence $\left(\theta_{k}\right)$ represents the arrival times of the market order, and the marks $\left(Z_{k}\right)$ valued in $\{+1,-1\}$ represent the side of the exchange with the convention:

- $Z_{k}=-1$ : trade exchanged at the best bid price, i.e. market sell order

- $Z_{k}=+1$ : trade exchanged at the best ask price, i.e. market buy order.

In this paper, we do not take into account the size of trades. Here is a an example of a database in a market where the tick size is 0.01 . To every trade, we attach on the same line the corresponding most recent quotes.

\begin{tabular}{c|c|c|c|c|} 
index $k$ & $\theta_{k}$ & Traded price & ASK-BID & $Z_{k}$ \\
\hline 1 & $09: 00: 01.123$ & 98.47 & $98.47-98.46$ & +1 \\
\hline 2 & $09: 00: 02.517$ & 98.46 & $98.47-98.46$ & -1 \\
\hline 3 & $09: 00: 02.985$ & 98.47 & $98.48-98.47$ & -1 \\
\hline 4 & $09: 00: 03.110$ & 98.47 & $98.48-98.47$ & -1 \\
\hline 5 & $09: 00: 05.458$ & 98.47 & $98.47-98.46$ & +1 \\
\hline
\end{tabular}

Let us now model the dependence between market order flow and stock price. We first look at the dependence between market order timestamps and stock price by considering that the counting process $\left(M_{t}\right)_{t}$ associated to $\left(\theta_{k}\right)$ is a Cox process with conditional intensity $\lambda\left(S_{t}\right)$, where we recall that $S_{t}$ is the time spent since the last price jump. This extends 
the simple case where $\left(M_{t}\right)$ is a Poisson process, and by assuming that $\lambda(s)$ is a decreasing function of $s$, this means that trade arrivals should occur less often in a period of price stability, i.e. when $S_{t}$ is large. We give a statistical procedure for the parametric estimation of the intensity function $\lambda$. The log-likelihood function associated to the counting process $\left(M_{t}\right)$ with intensity $\lambda\left(S_{t}\right)$ is given over $[0, T]$ by:

$$
\ln L_{T}=\int_{0}^{T} \ln \left[\lambda\left(S_{t}\right)\right] d M_{s}-\int_{0}^{T} \lambda\left(S_{t}\right) d t .
$$

Fix now $T=T_{n}$ the $n$-th jump time of the stock price. Since $S_{t}$ jumps at $\left\{T_{k}\right\}_{k=1, \ldots, n}$, so does $\lambda$. This implies that:

$$
\begin{aligned}
\int_{0}^{T} \lambda\left(S_{t}\right) d t=\sum_{k=1}^{n} \int_{T_{k-1}}^{T_{k}} \lambda\left(S_{t}\right) d t & =\sum_{k=1}^{n} \int_{T_{k-1}}^{T_{k}} \lambda\left(t-T_{k-1}\right) d t \\
& =\sum_{k=1}^{n} \int_{0}^{S_{k}} \lambda(t) d t .
\end{aligned}
$$

Moreoever, trades arrive at $\theta_{1}, \ldots, \theta_{m}$, and so:

$$
\int_{0}^{T} \ln \left[\lambda\left(S_{t}\right)\right] d M_{s}=\sum_{j=1}^{m} \ln \lambda\left(S_{\theta_{j}-}\right) .
$$

Putting all together, we have:

$$
\ln L_{T}=\sum_{j=1}^{m} \ln \lambda\left(S_{\theta_{j}-}\right)-\sum_{k=1}^{n} \int_{0}^{S_{k}} \lambda(t) d t .
$$

Assuming that $\lambda$ is of parametric form, we can then obtain the MLE estimator for $\lambda$ by maximizing over the parameters the above log-likelihood function. For example, we can use the following parametric forms, reproducing the intensity decay when $s$ is large:

$$
\lambda^{e x p}(s)=\lambda_{0}+a s^{r} e^{-k s}, \quad \lambda^{\text {pow }}(s)=\lambda_{0}+\frac{a s^{r}}{1+s^{k}}
$$

for $\lambda_{0}, a, r, k \geq 0$. Notice that in both cases,

- $\lambda$ is finite and smooth on $[0, \infty)$;

- when $a=0$ we fall into the homogeneous Poisson case of intensity $\lambda=\lambda_{0}$;

- when $r>0, \lambda(0)=\lambda(\infty)=\lambda_{0}$ with a maximum reached at $s_{*}>0$;

- for special value of $r$ and $k$ (e.g. $r \in \mathbb{N}$ in the exponential case), $\int_{0}^{s} \lambda(t) d t$ has an explicit form, leading to a much faster optimization.

In our case we have chosen:

$$
\lambda(s)=\lambda_{0}+a e^{-k s} \quad \text { and so } \quad \int_{0}^{s} \lambda(t) d t=\lambda_{0} s+a \frac{1-e^{-k s}}{k} .
$$


The estimated parameters on the data are:

\begin{tabular}{cc}
\hline$\lambda_{0}$ & 0.9772205 \\
$a$ & 77.0405310 \\
$k$ & 34.5428134 \\
\hline
\end{tabular}

Figure 2 shows the function $\lambda$ as a function of the quantile of $s$ in the distribution of $S$. For small renewal quantiles the function $\sigma^{2}(s)=h_{+}(s)+h_{-}(s)$, representing the jump intensity of the stock price no matter what direction, is big: in a similar fashion many trades arrive in the Limit Order Book when the price is not stable. On the contrary, when the price stabilizes, the trading activity is weak. Even if one might think that it is the trading activity which affects the stock price, we would like to stress that the goal of this model is to reproduce the correlation between the trading and the jump activity, without necessary explaining a cause-effect phenomenon.

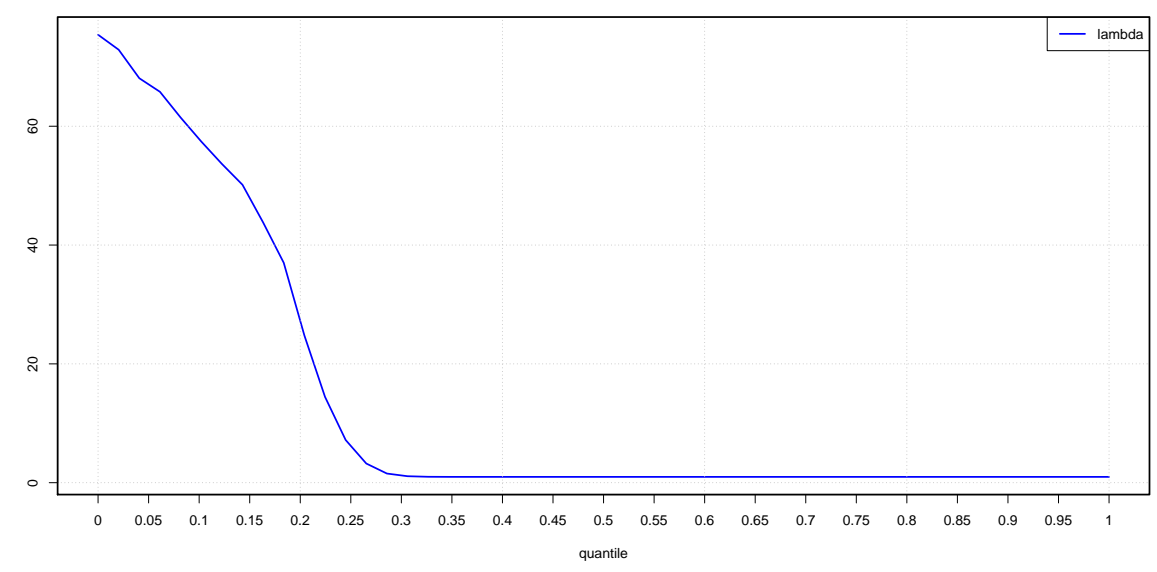

Figure 2: Estimation of $\lambda$ as a function of the renewal quantile

More generally, we shall consider an intensity function $\lambda$ in the form

$$
\lambda(s)=\lambda_{0}(s)+\lambda_{1}(s)
$$

where $\lambda_{0}$ is a bounded function on $\mathbb{R}_{+}$, and $\lambda_{1}$ is integrable on $\mathbb{R}_{+}$w.r.t. the Lebesgue measure, i.e. $\lambda_{1} \in L^{1}\left(\mathbb{R}_{+}\right)$. Let us define the function

$$
\mu(t, s)=\mathbb{E}\left[M_{t} \mid S_{0}=s\right]=\mathbb{E}\left[\int_{0}^{t} \lambda\left(S_{t}\right) \mid S_{0}=s\right], \quad t, s \geq 0 .
$$

The following result shows that $\mu$ is finite on $\mathbb{R}_{+} \times \mathbb{R}_{+}$.

Lemma 3.1. Under (3.1), we have

$$
\mu(t, s) \leq\left\|\lambda_{0}\right\|_{\infty} t+\left\|\lambda_{1}\right\|_{1}(1+\nu(t))<\infty, \quad \forall t, s \geq 0
$$


Proof. We first consider the case where $\lambda=\lambda_{0}$. It is then clear that $\mu(t, s) \leq\left\|\lambda_{0}\right\|_{\infty} t$. Next, consider the case where $\lambda=\lambda_{1}$. Set $\left(X_{k}=M_{T_{k}}-M_{T_{k-1}}\right)_{k \geq 1}$ the number of trades arrived between $T_{k-1}$ and $T_{k}$, with $T_{0}=0$ and $S_{0}=s$. Denoting by $\left(\mathcal{F}_{t}^{N}\right)$ the filtration generated by $\left(N_{t}\right)$, we obtain by the law of iterated conditional expectations:

$$
\begin{aligned}
\mu(t, s) & =\mathbb{E}\left[M_{t} \mid S_{0}=s\right]=\mathbb{E}\left[\sum_{k \geq 1} X_{k} 1_{T_{k} \leq t} \mid S_{0}=s\right] \\
& =\mathbb{E}\left[\sum_{k \geq 1} \mathbb{E}\left[X_{k} 1_{T_{k} \leq t} \mid \mathcal{F}_{t}^{N}\right] \mid S_{0}=s\right]=\mathbb{E}\left[\sum_{k \geq 1} 1_{T_{k} \leq t} \mathbb{E}\left[X_{k} \mid \mathcal{F}_{t}^{N}\right] \mid S_{0}=s\right] \\
& =\mathbb{E}\left[\sum_{k \geq 1} 1_{T_{k} \leq t} \mathbb{E}\left[\int_{T_{k-1}}^{T_{k}} \lambda_{1}\left(S_{u}\right) d u\right] \mid S_{0}=s\right] \\
& =\mathbb{E}\left[\sum_{k \geq 1} 1_{T_{k} \leq t} \mathbb{E}\left[\int_{0}^{T_{k}-T_{k-1}} \lambda_{1}\left(u+s 1_{k=1}\right) d u\right] \mid S_{0}=s\right] \\
& \leq\left\|\lambda_{1}\right\|_{1} \mathbb{E}\left[\sum_{k \geq 1} 1_{T_{k} \leq t} \mid S_{0}=s\right]=\left\|\lambda_{1}\right\|_{1} \nu(t, s) \leq\left\|\lambda_{1}\right\|_{1}(1+\nu(t)) .
\end{aligned}
$$

By combining the two cases when $\lambda=\lambda_{0}+\lambda_{1}$, we get the required bound for $\mu$.

We next consider the dependence between market order trade and stock price in the limit order book (LOB) through the following relation:

$$
Z_{k}=\Gamma_{k} I_{\theta_{k}-}
$$

where $\left(\Gamma_{k}\right)_{k}$ is an i.i.d. sequence, independent of all other processes, and distributed according to a Bernoulli law on $\{+1,-1\}$ with parameter $(1+\rho) / 2$, with $\rho \in[-1,1]$. In this case, we easily see that

$$
\rho=\operatorname{Corr}\left(Z_{k}, I_{\theta_{k}^{-}}\right)
$$

so that the parameter $\rho$ has the following interpretation:

- $\rho=0$ : trade sides do not depend on the stock price, and market order flow arrive independently at best bid and best ask. This is the usual assumption made in the literature, see e.g. [5].

- $\rho>0$ : most of the trade sides are concordant with the direction of the last jump of the stock price: Market orders arrive more often in the strong side $(+)$ of the LOB, that is the side in the same direction than the last jump, i.e. best ask (resp. bid) when price jumped upwards (resp. downwards).

- $\rho<0$ : most of the trade sides are discordant with the direction of the last jump of the stock price: Market orders arrive more often in the weak side $(-)$ of the LOB, that is the side in the opposite direction than the last jump, i.e. best bid (resp. ask) when price jumped upwards (resp. downwards). 
From (3.2), and by the strong law of large numbers, we have a consistent estimator of $\rho$ given by:

$$
\hat{\rho}^{(n)}=\frac{1}{n} \sum_{k=1}^{n} \frac{Z_{k}}{I_{\theta_{k}-}}=\frac{1}{n} \sum_{k=1}^{n} Z_{k} I_{\theta_{k}^{-}} \longrightarrow \mathbb{E}\left[\Gamma_{k}\right]=\rho, \text { a.s. },
$$

as $n$ goes to infinity. Estimation on real data leads to a value of $\rho$ around $-50 \%$. This means that about 3 over 4 trades arrive on the weak side of the LOB. Recall that stock price usually exhibits a short-term mean reversion, i.e. a negative correlation $\alpha$ of price increments, so that

$$
\alpha \rho>0 \text {. }
$$

The fact that $\alpha$ and $\rho$ are of the same sign is consistent with the adverse selection principle. Indeed, suppose on the contrary that $\alpha$ and $\rho$ are of opposite sign, say $\alpha<0$ and $\rho>0$, and assume e.g. that the last price jumped downwards. Then $\rho>0$ means that most of the trade will occur at best bid, hence will execute limit buy order in a bull market (since $\alpha<0$ ), hence will allow market makers on best bid to open a low risk profitable position, contradicting the adverse selection principle. The parameter $\rho$ is a measure of the adverse selection: the larger is the positive value $\alpha \rho$, the larger is the adverse selection risk.

We define the concordant $(+)$ and discordant (-) trade intensity as:

$$
\lambda_{ \pm}(s):=\left(\frac{1 \pm \rho}{2}\right) \lambda(s)
$$

This definition is coherent with the definition of $h_{ \pm}(s)$, where \pm indexes the concordance $(+)$ or discordance (-) of two consecutive jumps.

\section{The market making problem}

The agent strategy consists in placing continuously limit orders of small size $L \in \mathbb{N} \backslash\{0\}$ w.r.t. to the total liquidity provided by all the market makers, at the best price available, while respecting an inventory constraint. The market making strategy control is then described by a pair of predictable processes $\left(\ell^{+}, \ell^{-}\right)$valued in $\{0,1\}$ with the convention that when $\ell_{t}^{+}=1$ (resp. $\ell_{t}^{-}=1$ ), this means that a limit order of fixed size $L$ is posted at time $t$ on the strong (resp. weak) side, i.e. on the side $+I_{t^{-}}\left(\operatorname{resp} .-I_{t^{-}}\right)$, and when $\ell_{t}^{ \pm}=$ 0 , no limit order is submitted.

Every time that a market order arrives in the LOB (according to the mechanism described in Section 3), and the agent has already sent a limit order on the corresponding side, she is executed according to a random variable, which depends on the LOB rule:

- Price time priority (PTP): in a PTP order book, orders are matched according to their price (more priority to those closer to the mid price) and time (like in single file queue). 
- Prorata: in a (pure) prorata order book, there is price prioriy (as in PTP), but not the time one. Multiple market makers are executed when a single trade arrives, according to a proportion (evaluated deterministically) rewarding the limit orders of bigger size.

Notice that, if the price jumps upwards and the agent is in the condition to place her limit orders immediately after the jump, she will find a huge volume of concurrent limit order on the ask side (old orders), while very few on the bid side. The situation is symmetric when the price has jumped downwards. This leads us to have, in both cases (PTP and prorata), the existence of distributions:

$$
\vartheta_{L}^{ \pm}(d k) \text { on }\{0, \ldots, L\}
$$

where $\vartheta_{L}^{+}(d k)$ (resp. $\left.\vartheta_{L}^{-}(d k)\right)$ is the distribution of the executed quantity of limit order of size $L$ in the concordant (resp. discordant), i.e. strong (resp. weak) side, of the LOB. Once again, the sign \pm indexing the distribution must not be interpreted as the ask/bid side of the LOB but as the strong/weak side of the LOB taking into account the last price direction.

Remark 4.1. Unfortunately, the agent execution cannot be estimated before playing or backtesting a zero intelligence strategy, by placing continuously limit orders on both sides of the limit order book. This problem cannot be overcome. So far we have suggested this approach since it reflects a certain philosophy: the flow of market trades can be easily studied consulting on a database of historical data. Furthermore, because of the number of market trades, statistics are significant. Execution is much more delicate, but thought as stable in time.

Moreoever, in order to be coherent with the small agent assumption (the price is exogenous and not impacted by the agent strategy), we assume that, if the price jumps crossing the level of a limit order placed by the agent, the latter is automatically executed. This can be justified by saying that, since the agent is small, the price jumps independently of the presence of her limit order. Futhermore, since the spread is constantly one tick and orders are placed at the best price, when the price jumps, limit orders converts automatically to market ones, which are immediately executed.

We also assume that each transaction is subject to a fixed $\operatorname{cost} \varepsilon \geq 0$. Let us then denote by $\left(X_{t}\right)$ and $\left(Y_{t}\right)$ the cash and inventory process of the agent, valued respectively in $\mathbb{R}$ and $\mathbb{Z}$.

Lemma 4.1. For a market making strategy $\left(\ell^{+}, \ell^{-}\right)$, the dynamics of the portfolio value processes $X$ and $Y$ are given by

$$
\begin{aligned}
d X_{t}= & +\int k \ell_{t-}^{+}\left[+P_{t-} I_{t-}+\delta-\varepsilon\right]\left[R_{t r d}^{+}\left(d t, d k, S_{t-}\right)+R_{j m p}^{+}\left(d t, d k, S_{t-}\right)\right] \\
& +\int k \ell_{t-}^{-}\left[-P_{t-} I_{t-}+\delta-\varepsilon\right]\left[R_{t r d}^{-}\left(d t, d k, S_{t-}\right)+R_{j m p}^{-}\left(d t, d k, S_{t-}\right)\right] \\
d Y_{t}= & -\int k \ell_{t-}^{+} I_{t-}\left[R_{t r d}^{+}\left(d t, d k, S_{t-}\right)+R_{j m p}^{+}\left(d t, d k, S_{t-}\right)\right] \\
& +\int k \ell_{t-}^{-} I_{t-}\left[R_{t r d}^{-}\left(d t, d k, S_{t-}\right)+R_{j m p}^{-}\left(d t, d k, S_{t-}\right)\right]
\end{aligned}
$$


where $R_{t r d}^{ \pm}=R_{t r d}^{ \pm}\left(d t, d k, S_{t-}\right)$ is a random measure with intensity measure:

$$
\lambda_{ \pm}\left(S_{t^{-}}\right) d t \otimes \vartheta_{L}^{ \pm}(d k)
$$

while $R_{j m p}^{ \pm}=R_{j m p}^{ \pm}\left(d t, d k, S_{t-}\right)$ has intensity measure:

$$
h_{ \pm}\left(S_{t^{-}}\right) d t \otimes \delta_{L}(d k),
$$

where $\delta_{L}$ is the Dirac measure at $L$.

Proof. To fix the ideas, assume that, at time $t, i=I_{t-}=+1$, or equivalently the last jump of the price has been upwards (in the case $i=-1$ the proof is symmetric). Then if $\ell^{ \pm}=1$, i.e. if the agent has placed a limit order on the strong (i.e. ask) or the weak (i.e. bid) side:

- either she can be executed by an incoming trade of a random number of quantities $k$, where $k$ has distribution $\vartheta_{L}^{ \pm}$, with trade intensity $\lambda_{ \pm}(s)$

- or she can be entirely executed, thus $k=L$, by a jump of the price, with rate $h_{ \pm}(s)$.

In both cases, the agent inventory $Y$ jumps of $\mp k$, while her wealth $X$ jumps of $k$ times $\pm P_{t-}$ (asset sold or bought), plus the market making prime of $\delta$ (the half spread), minus the fixed $\operatorname{cost} \varepsilon$.

As a consequence of the above lemma, we see that given a market making control $\ell$ $=\left(\ell^{+}, \ell^{-}\right)$, the infinitesimal generator of the controlled Markov system $\left(P_{t}, I_{t}, S_{t}, X_{t}, Y_{t}\right)$ is given by:

$$
\frac{\partial \varphi}{\partial s}+\mathcal{L}\left[\ell^{+}, \ell^{-}\right] \varphi
$$

with

$$
\mathcal{L}\left[\ell^{+}, \ell^{-}\right]=\mathcal{L}_{\text {trd }}^{+}\left[\ell^{+}\right]+\mathcal{L}_{j m p}^{+}\left[\ell^{+}\right]+\mathcal{L}_{\text {trd }}^{-}\left[\ell^{-}\right]+\mathcal{L}_{j m p}^{-}\left[\ell^{-}\right]
$$

where for a test function $\varphi(p, i, s, x, y)$ :

$$
\begin{aligned}
\mathcal{L}_{\text {trd }}^{ \pm}[\ell] \varphi & =\lambda_{ \pm}(s) \int[\varphi(t, p, i, s, x+\ell k( \pm i p+\delta-\epsilon), y \mp i \ell k)-\varphi] \vartheta_{L}^{ \pm}(d k) \\
\mathcal{L}_{j m p}^{ \pm}[\ell] \varphi & =h_{ \pm}(s)[\varphi(t, p \pm 2 \delta i, \pm i, 0, x+\ell L( \pm i p+\delta-\epsilon), y \mp i \ell L)-\varphi] .
\end{aligned}
$$

Notice that, a priori, $Y_{t} \in \mathbb{Z}$. We call $\mathbb{Y}$ the space of admissible inventory. If the trader is allowed to have an unbounded inventory, $\mathbb{Y}=\mathbb{Z}$, otherwise we can reduce it to:

$$
\mathbb{Y}=\left\{-y_{\max }, \ldots, y_{\max }\right\}
$$

where $y_{\max }$ is a finite integer chosen by the agent herself, and we say that a market making control $\ell=\left(\ell^{+}, \ell^{-}\right)$is admissible, denoted $\ell \in \mathcal{A}$, if the associated inventory process remains valued in $\mathbb{Y}$. 
We now consider the following criterion for the market making optimization problem, as usually adopted in [5], [9], [15]: the agent is looking for the optimal admissible market making strategy, which maximizes her expected

$$
P N L_{T}=X_{T}+Y_{T} P_{T}
$$

representing her portfolio value at terminal date $T$, evaluated at the mid price, and has to liquidate her terminal inventory by a market order at the cost:

$$
\operatorname{Close}\left(Y_{T}\right)=(\delta+\varepsilon)\left|Y_{T}\right| .
$$

Moreover, she is penalized when holding inventory $Y$ during the trading $[0, T]$ through the term:

$$
\operatorname{Risk}_{0, T}=\eta \int_{0}^{T} Y_{t}^{2} d[P]_{t}
$$

where $\eta \geq 0$ is a positive parameter related to the risk aversion of the agent, and $[P]$ is the quadratic variation of the mid price process $P$. From the definition (2.1) of the pure jump process $P$, we have:

$$
[P]_{t}=4 \delta^{2} \sum_{i=1}^{N_{t}} J_{k}^{2}=4 \delta^{2} N_{t},
$$

whose dual predictable projection (also called sharp bracket) $\langle P\rangle$ is given by:

$$
d\langle P\rangle_{t}=4 \delta^{2} \sigma^{2}\left(S_{t}\right) d t
$$

by recalling that $\sigma^{2}\left(S_{t}\right)$ is the intensity of $N_{t}$, see Remark 2.1 .

We can now define in our MRP model the value function associated to the market making problem:

$$
v(t, p, i, s, x, y)=\max _{\ell \in \mathcal{A}} \mathbb{E}_{t, p, i, s, x, y}\left[X_{T}+Y_{T} P_{T}-(\delta+\epsilon)\left|Y_{T}\right|-\bar{\eta} \int_{t}^{T} Y_{u}^{2} \sigma^{2}\left(S_{u}\right) d u\right],
$$

where $\bar{\eta}=4 \delta^{2} \eta, \mathbb{E}_{t, p, i, s, x, y}$ denotes the expectation operator under the initial conditions $P_{t}=$ $p, I_{t}=i, S_{t}=s, X_{t}=x, Y_{t}=y$, for $(t, p, i, s, x, y) \in[0, T] \times\left(P_{0}+2 \delta \mathbb{Z}\right) \times\{-1,1\} \times \mathbb{R}_{+} \times \mathbb{R} \times \mathbb{Y}$.

In the sequel, we shall assume that either the admissible inventory set $\mathbb{Y}$ is bounded as in (4.2), or the inventory penalization parameter $\eta$ is strictly positive. We state some estimate bounds on the value function.

Proposition 4.1. The value function is bounded by:

$$
x+y p+\omega_{*}(t, s, y) \leq v(t, p, i, s, x, y) \leq x+y p+\omega^{*}(t, s),
$$

for all $(t, p, i, s, x, y) \in[0, T] \times\left(P_{0}+2 \delta \mathbb{Z}\right) \times\{-1,1\} \times \mathbb{R}_{+} \times \mathbb{R} \times \mathbb{Y}$, where

$$
\begin{aligned}
\omega_{*}(t, s, y) & =-(\delta+\varepsilon)|y|-2 \delta\left(|y|+2 \delta \eta y^{2}\right) \nu(T-t, s), \\
\omega^{*}(t, s) & =\left\{\begin{array}{cl}
L \delta[\mu(T-t, s)+\nu(T-t, s)]+2 \delta y_{\max } \nu(T-t, s), & \text { if } \mathbb{Y} \text { is bounded } \\
L \delta[\mu(T-t, s)+\nu(T-t, s)]+\frac{1}{4 \eta} \nu(T-t, s), & \text { if } \eta>0 .
\end{array}\right.
\end{aligned}
$$


Proof. By considering the strategy consisting in holding the inventory until the end of the trading day, we have the lower bound:

$$
\begin{aligned}
v(t, p, i, s, x, y) & \geq \mathbb{E}_{t, p, i, s, x, y}\left[X_{T}+Y_{T} P_{T}-(\delta+\varepsilon)\left|Y_{T}\right|-\bar{\eta} \int_{t}^{T} Y_{u}^{2} \sigma^{2}\left(S_{u}\right) d u\right] \\
& =x+y p+y \mathbb{E}_{t, p, i, s}\left[P_{T}-p\right]-(\delta+\varepsilon)|y|-4 \delta^{2} \eta y^{2} \mathbb{E}_{t, s}\left[\int_{t}^{T} \sigma^{2}\left(S_{u}\right) d u\right] \\
& =x+y p+y \mathbb{E}_{t, p, i, s}\left[P_{T}-p\right]-(\delta+\varepsilon)|y|-4 \delta^{2} \eta y^{2} \nu(T-t, s) .
\end{aligned}
$$

Moreover, we have

$$
\begin{aligned}
y \mathbb{E}_{t, p, i, s}\left[P_{T}-p\right] & \geq-2 \delta|y|\left|\mathbb{E}_{t, p, i, s}\left[\sum_{k=N_{t}+1}^{N_{T}} J_{k}\right]\right| \\
& \geq-2 \delta|y| \mathbb{E}\left[N_{T}-N_{t} \mid S_{t}=s\right]=-2 \delta|y| \nu(T-t, s),
\end{aligned}
$$

which shows the lower bound for the value function $v$.

About the upper bound, we see by Itô's formula to the product $Y_{t} P_{t}$ that for any admissible strategy $\ell \in \mathcal{A}$ :

$$
\begin{aligned}
& \mathbb{E}_{t, p, i, s, x, y}\left[X_{T}+Y_{T} P_{T}-(\delta+\epsilon)\left|Y_{T}\right|-\bar{\eta} \int_{t}^{T} Y_{u}^{2} \sigma^{2}\left(S_{u}\right) d u\right] \\
\leq & x+y p+\mathbb{E}_{t, p, i, s, x, y}\left[\int_{t}^{T}\left(d X_{u}+P_{u^{-}} d Y_{u}\right)+\int_{t}^{T}\left(Y_{u^{-}} d P_{u}-\eta Y_{u}^{2} d<P>_{u}\right)\right] \\
= & x+y p+\mathbb{E}_{t, p, i, s, x, y}\left[\int_{t}^{T} d Z_{u}^{1}+\int_{t}^{T} d Z_{u}^{2}\right],
\end{aligned}
$$

where we set $d Z_{t}^{1}=d X_{t}+P_{t^{-}} d Y_{t}$, and $d Z_{t}^{2}=Y_{t^{-}} d P_{t}-\eta Y_{t}^{2} d<P>_{t}$. From the dynamics of $X$ and $Y$ in Lemma 4.1, we have:

$$
\begin{aligned}
\mathbb{E}_{t, p, i, s, x, y}\left[\int_{t}^{T} d Z_{u}^{1}\right] \leq & \delta \mathbb{E}_{t, s}\left[\int_{t}^{T} \int k \ell_{t-}^{+}\left[R_{t r d}^{+}\left(d u, d k, S_{u-}\right)+R_{j m p}^{+}\left(d u, d k, S_{u-}\right)\right]\right] \\
& +\delta \mathbb{E}_{t, s}\left[\int_{t}^{T} k \ell_{t-}^{-}\left[R_{t r d}^{-}\left(d u, d k, S_{u-}\right)+R_{j m p}^{-}\left(d u, d k, S_{u-}\right)\right]\right] \\
\leq & L \delta \mathbb{E}_{t, s}\left[\int_{t}^{T} \int R_{t r d}^{+}\left(d u, d k, S_{u-}\right)+R_{t r d}^{-}\left(d u, d k, S_{u-}\right)\right] \\
& \quad+L \delta \mathbb{E}_{t, s}\left[\int_{t}^{T} \int R_{j m p}^{+}\left(d u, d k, S_{u-}\right)+R_{j m p}^{-}\left(d u, d k, S_{u-}\right)\right] \\
= & L \delta\left(\mathbb{E}_{t, s}\left[\int_{t}^{T} \lambda_{+}\left(S_{u}\right)+\lambda_{-}\left(S_{u}\right) d u\right]+\mathbb{E}_{t, s}\left[\int_{t}^{T} h_{+}\left(S_{u}\right)+h_{-}\left(S_{u}\right) d u\right]\right) \\
= & L \delta(\mu(T-t, s)+\nu(T-t, s)) .
\end{aligned}
$$

On the other hand, for the estimate on $Z^{2}$, we distinguish the cases whether $\mathbb{Y}$ is bounded or $\eta>0$. When $\mathbb{Y}$ is bounded, i.e. $\mathbb{Y}=\left\{-y_{\max }, \ldots, y_{\max }\right\}$, we have:

$$
\begin{aligned}
\mathbb{E}_{t, p, i, s, x, y}\left[\int_{t}^{T} d Z_{u}^{2}\right] & \leq \mathbb{E}_{t, p, i, s, x, y}\left[\int_{t}^{T} Y_{u^{-}} d P_{u}\right]=2 \delta \mathbb{E}_{t, p, i, s, x, y}\left[\sum_{k=N_{t}+1}^{N_{T}} Y_{T_{k-1}}\right] \\
& \leq 2 \delta y_{\max } \mathbb{E}\left[N_{T}-N_{t} \mid S_{t}=s\right]=2 \delta y_{\max } \nu(T-t, s) .
\end{aligned}
$$


Otherwise, when $\eta>0$, we have:

$$
\begin{aligned}
\mathbb{E}_{t, p, i, s, x, y}\left[\int_{t}^{T} d Z_{u}^{2}\right] & =\mathbb{E}_{t, p, i, s, x, y}\left[\int_{t}^{T}\left(Y_{u^{-}} d P_{u}-\eta Y_{u}^{2} d[P]_{u}\right)\right] \\
& =\mathbb{E}_{t, p, i, s, x, y}\left[\sum_{k=N_{t}+1}^{N_{T}} Y_{T_{k-1}}\left(P_{T_{k}}-P_{T_{k-1}}\right)-\eta Y_{T_{k-1}}^{2}\left(P_{T_{k}}-P_{T_{k-1}}\right)^{2}\right] \\
& \leq \frac{1}{4 \eta} \mathbb{E}\left[N_{T}-N_{t} \mid S_{t}=s\right]=\frac{1}{4 \eta} \nu(T-t, s)
\end{aligned}
$$

where we used the fact that $z-\eta z^{2} \leq \frac{1}{4 \eta}$ for all $z \in \mathbb{R}$. By plugging (4.5), (4.6) or (4.7) into (4.4), we get the required upper bound for the value function.

Corollary 4.1. Under (3.1), we have the following growth condition on the value function: there exists some positive constant $C$ s.t.

$$
x+y p-C\left(1+y^{2}\right) \leq v(t, p, i, s, x, y) \leq x+y p+C,
$$

for all $(t, p, i, s, x, y) \in[0, T] \times\left(P_{0}+2 \delta \mathbb{Z}\right) \times\{-1,1\} \times \mathbb{R}_{+} \times \mathbb{R} \times \mathbb{Y}$.

Proof. Under (3.1), by Lemma 2.1, and Lemma 3.1, we have for all $(t, s) \in[0, T] \times \mathbb{R}_{+}$:

$$
\begin{aligned}
\nu(T-t, s) & \leq 1+\nu(T), \\
\mu(T-t, s) & \leq\left\|\lambda_{0}\right\|_{\infty} T+\left\|\lambda_{1}\right\|_{1}(1+\nu(T)),
\end{aligned}
$$

since $\nu(t)$ is a nondecreasing function in $t$. We conclude with the estimates in Proposition 4.1 .

\section{Resolution of the market making problem}

\subsection{Variable reduction and analytical characterization}

In this paragraph, by exploiting the structure and symmetry of our model w.r.t. the strong/weak side of the LOB, we show that the value function $v$ defined in (4.3), and involving a priori 6 state variables, can be reduced to a function of 3 variables, that we characterize in terms of solution to a system of integro-ordinary differential equations.

Theorem 5.1. Under (3.1), the value function $v$ is given by:

$$
v(t, p, i, s, x, y)=x+y p+\omega(t, s, y i)
$$

where $\omega=\omega(t, s, q)$ defined on the domain $[0, T] \times \mathbb{R}_{+} \times \mathbb{Y}$ is the unique viscosity solution with quadratic growth in $y$ to:

$$
\begin{gathered}
\left.\left.+\max _{\ell \in\{0,1\}, q-\ell L \in \mathbb{Y}}\left(\mathcal{N}_{t r d}^{+}[\ell]+\mathcal{N}_{j m p}^{+}[\ell]\right) \omega+\max _{\ell \in\{0,1\}, q+\ell L \in \mathbb{Y}}\left(\partial_{s}-\kappa(s)\right] \omega+c(s) q-\bar{\eta} \sigma^{2}(s) q^{2}-1 \ell\right]+\mathcal{N}_{j m p}^{-}[\ell]\right) \omega=0, \\
\omega(T, s, q)=-|q|(\delta+\varepsilon),
\end{gathered}
$$


where

$$
c(s):=2 \delta\left[h_{+}(s)-h_{-}(s)\right], \quad \kappa(s):=\lambda(s)+\sigma^{2}(s),
$$

and the operators $\mathcal{N}$ 's are given by:

$$
\begin{aligned}
\mathcal{N}_{t r d}^{ \pm}[\ell] \omega & :=\lambda_{ \pm}(s) \int[\omega(t, s, q \mp k \ell)+k \ell(+\delta-\varepsilon)] \vartheta_{L}^{ \pm}(d k) \\
\mathcal{N}_{j m p}^{ \pm}[\ell] \omega: & =h_{ \pm}(s)[\omega(t, 0, \pm q-L \ell)+L \ell(-\delta-\varepsilon)] .
\end{aligned}
$$

Proof. By classical stochastic optimal control (see e.g. [18]), it is known that $v$ is the unique viscosity solution satisfying the growth condition (4.8) to:

$$
\begin{aligned}
\partial_{t} v+\partial_{s} v+\max _{\ell^{ \pm} \in\{0,1\}, y \mp i \ell^{ \pm} L \in \mathbb{Y}} \mathcal{L}\left[\ell^{+}, \ell^{-}\right] v-\bar{\eta} \sigma^{2}(s) y^{2} & =0 \\
v(T, p, i, s, x, y) & =x+y p-|y|(\delta+\varepsilon) .
\end{aligned}
$$

We now make the first ansatz to remove the variables $x$ and $p$ :

$$
v(t, p, i, s, x, y)=x+y p+w(t, s, y, i) .
$$

From the expression of the infinitesimal generator $\mathcal{L}\left[\ell^{+}, \ell^{-}\right]$in $(4.1)$, and using this ansatz, we get:

$$
\begin{aligned}
\mathcal{L}_{t r d}^{ \pm}[\ell] v & =\lambda_{ \pm}(s) \int[(\delta-\varepsilon) \ell k+w(t, s, y \mp i \ell k, i)-w] \vartheta_{L}^{ \pm}(d k), \\
\mathcal{L}_{j m p}^{ \pm}[\ell] v & =h_{ \pm}(s)[(-\delta-\varepsilon) \ell L \pm 2 \delta i y+w(t, 0, y \mp i \ell L, \pm i)-w]
\end{aligned}
$$

which leads by summing up:

$$
\mathcal{L}[\ell] v=\mathcal{M}_{t r d}^{+}\left[\ell^{+}\right] w+\mathcal{M}_{t r d}^{-}\left[\ell^{-}\right] w+\mathcal{M}_{j m p}^{+}\left[\ell^{+}\right] w+\mathcal{M}_{j m p}^{-}\left[\ell^{-}\right] w-\kappa(s) w+c(s) i y,
$$

where $c$ and $\kappa$ are given in (5.4), and

$$
\begin{aligned}
\mathcal{M}_{t r d}^{ \pm}[\ell] w & :=\lambda_{ \pm}(s) \int[(\delta-\varepsilon) \ell k+w(t, s, y \mp i \ell k, i)] \vartheta_{L}^{ \pm}(d k) \\
\mathcal{M}_{j m p}^{ \pm}[\ell] w & :=h_{ \pm}(s)[(-\delta-\varepsilon) \ell L+w(t, 0, y \mp i \ell L, \pm i)] .
\end{aligned}
$$

Since $\partial_{t} v=\partial_{t} w$, and $\partial_{s} v=\partial_{s} w$, the HJB equation (5.6)-(5.7) becomes:

$$
\begin{aligned}
& {\left[\partial_{t}+\partial_{s}-\kappa(s)\right] w+c(s) y i-\bar{\eta} \sigma^{2}(s) y^{2} } \\
+ & \max _{\ell \in\{0,1\}: y-i \ell L \in \mathbb{Y}}\left(\mathcal{M}_{t r d}^{+}[\ell] w+\mathcal{M}_{j m p}^{+}[\ell] w\right) \\
+\max _{\ell \in\{0,1\}: y+i \ell L \in \mathbb{Y}}\left(\mathcal{M}_{t r d}^{-}[\ell] w+\mathcal{M}_{j m p}^{-}[\ell] w\right) & =0 \\
w(T, s, y, i) & =-|y|(\delta+\varepsilon)
\end{aligned}
$$

in the domain $[0, T] \times \mathbb{R}_{+} \times \mathbb{Y} \times\{ \pm\}$. Next, we remove another variable by the change of variable $q=y i$, with the ansatz:

$$
w(t, s, y, i)=\omega(t, s, y i)
$$


and by noting that $i^{2}=1$, so that:

$$
\begin{gathered}
\mathcal{M}_{t r d}^{ \pm}[\ell] w=\mathcal{N}_{t r d}^{ \pm}[\ell] \omega:=\lambda_{ \pm}(s) \int[(\delta-\varepsilon) \ell k+\omega(t, s, q \mp \ell k)] \vartheta_{L}^{ \pm}(d k) \\
\mathcal{M}_{j m p}^{ \pm}[\ell] w=\mathcal{N}_{j m p}^{ \pm}[\ell] \omega:=h_{ \pm}(s)[(-\delta-\varepsilon) \ell L+\omega(t, 0, \pm q-\ell L)] .
\end{gathered}
$$

Finally, by noting that $\partial_{t} w=\partial_{t} \omega, \partial_{s} w=\partial_{s} \omega$, and $y \mp i \ell L \in \mathbb{Y}$ iff $q \mp \ell L \in \mathbb{Y}$, the HJB equation (5.8)-(5.9) becomes:

$$
\begin{aligned}
& {\left[\partial_{t}+\partial_{s}-\kappa(s)\right] \omega+c(s) q-\bar{\eta} \sigma^{2}(s) q^{2}} \\
& +\max _{\ell \in\{0,1\}, q-\ell L \in \mathbb{Y}}\left(\mathcal{N}_{t r d}^{+}[\ell]+\mathcal{N}_{j m p}^{+}[\ell]\right) \omega \\
& +\max _{\ell \in\{0,1\}, q+\ell L \in \mathbb{Y}}\left(\mathcal{N}_{t r d}^{-}[\ell]+\mathcal{N}_{j m p}^{-}[\ell]\right) \omega=0 \\
& \omega(T, s, q)=-|q|(\delta+\varepsilon)
\end{aligned}
$$

in the domain $[0, T] \times \mathbb{R}_{+} \times \mathbb{Y}$. This ends the proof.

The above theorem states that the solution (value function and optimal control) of the market making problem are completely characterized in terms of integral equation involving three observable state variables: the current time $t$, the elapsed time $s$ from last price jump, and the strong inventory $q=y i$. In the next paragraph, we give a numerical scheme for computing the value function and optimal market making strategies.

\section{$5.2 \quad$ Numerical scheme}

We provide in this paragraph a numerical scheme for the solution $\omega(t, s, q)$ to the integro ODE (5.2)-(5.3) in the case $\mathbb{Y}=\left\{-y_{\max }, \ldots,+y_{\max }\right\}$. Actually, it is more natural and convenient to work in the domain $[0,1)$ of the quantile of the elapsed time rather than the domain $\mathbb{R}_{+}$of its absolute value. Denote by $\mathcal{Q}:=F^{-1}$ the quantile function of the renewal distribution function $F$ in (2.2), which has density

$$
f=\frac{1+\alpha}{2} f_{+}+\frac{1-\alpha}{2} f_{-}
$$

and set $\hat{g}:=g \circ \mathcal{Q}$, for any function $g:[0, \infty) \rightarrow \mathbb{R}$. Let us now make the change of variable: $\hat{s}:=F(s)$, and define $\hat{\omega}(t, \hat{s}, q):=\omega(t, \mathcal{Q}(s), q)$ as a function of the quantile of the elapsed time. Since $\mathcal{Q}(0)=0$ and

$$
\frac{\partial}{\partial s}=\frac{\partial \hat{s}}{\partial s} \frac{\partial}{\partial \hat{s}}=f(s) \frac{\partial}{\partial \hat{s}}=f[\mathcal{Q}(\hat{s})] \frac{\partial}{\partial \hat{s}}=\hat{f}(\hat{s}) \frac{\partial}{\partial \hat{s}},
$$

we immediately see that $\hat{\omega}$ is solution to the integro ODE on $[0, T] \times[0,1) \times \mathbb{Y}$ :

$$
\begin{aligned}
& {\left[\partial_{t}+\hat{f}(\hat{s}) \partial_{\hat{s}}-\hat{\kappa}(\hat{s})\right] \hat{\omega}+\hat{c}(\hat{s}) q-\bar{\eta} \hat{\sigma}^{2}(\hat{s}) q^{2}} \\
& \quad+\max _{\ell \in\{0,1\}, q-\ell L \in \mathbb{Y}}\left(\hat{\mathcal{N}}_{t r d}^{+}[\ell]+\hat{\mathcal{N}}_{j m p}^{+}[\ell]\right) \hat{\omega} \\
& \quad+\max _{\ell \in\{0,1\}, q+\ell L \in \mathbb{Y}}\left(\hat{\mathcal{N}}_{t r d}^{-}[\ell]+\hat{\mathcal{N}}_{j m p}^{-}[\ell]\right) \hat{\omega}=0 \\
& \hat{\omega}(T, s, q)=-|q|(\delta+\varepsilon)
\end{aligned}
$$


on $[0, T] \times[0,1) \times \mathbb{Y}$, where the operators $\hat{\mathcal{N}}^{\prime}$ 's are given by:

$$
\begin{aligned}
\hat{\mathcal{N}}_{t r d}^{ \pm}[\ell] \hat{\omega} & :=\hat{\lambda}_{ \pm}(\hat{s}) \int[\hat{\omega}(t, \hat{s}, q \mp k \ell)+k \ell(+\delta-\varepsilon)] \vartheta_{L}^{ \pm}(d k) \\
\hat{\mathcal{N}}_{j m p}^{ \pm}[\ell] \hat{\omega} & :=\hat{h}_{ \pm}(\hat{s})[\hat{\omega}(t, 0, \pm q-L \ell)+L \ell(-\delta-\varepsilon)] .
\end{aligned}
$$

Given a uniform discretization grid $\left\{\left(t_{n}, \hat{s}_{i}\right)\right\}_{n=0, \ldots, N}^{i=0, \ldots, M}$ with step $\Delta t \times \Delta \hat{s}$ on $[0, T] \times[0,1]$, we provide an approximation

$$
\hat{\omega}_{i, q}^{n} \quad \approx \hat{\omega}\left(t=t_{n}, \hat{s}=\hat{s}_{i}, q\right) ; \quad 0 \leq i<M, \quad 0 \leq n \leq N, \quad q \in \mathbb{Y},
$$

with the discretization scheme:

$$
\begin{aligned}
\frac{\hat{\omega}_{i, q}^{n+1}-\hat{\omega}_{i, q}^{n}}{\Delta t}+\hat{f} & (\hat{s}) \frac{\hat{\omega}_{i+1, q}^{n}-\hat{\omega}_{i, q}^{n}}{\Delta s}-\hat{\kappa}\left(\hat{s}_{i}\right) \hat{\omega}_{i, q}^{n}+\hat{c}\left(\hat{s}_{i}\right) q-\bar{\eta} \hat{\sigma}^{2}\left(\hat{s}_{i}\right) q^{2} \\
& +\max _{\ell \in\{0,1\}:|q-\ell L| \in \mathbb{Y}}\left[\left(\hat{N}_{\text {trd }}^{+}[l]\right)_{i, q}^{n+1}+\left(\hat{N}_{j m p}^{+}[l]\right)_{i, q}^{n+1}\right] \\
& +\max _{\ell \in\{0,1\}:|q+\ell L| \in \mathbb{Y}}\left[\left(\hat{N}_{\text {trd }}^{-}[l]\right)_{i, q}^{n+1}+\left(\hat{N}_{j m p}^{-}[l]\right)_{i, q}^{n+1}\right]=0
\end{aligned}
$$

where:

$$
\begin{aligned}
\left(\hat{N}_{\text {trd }}^{+}[0]\right)_{i, q}^{n+1} & =\hat{\lambda}_{ \pm}\left(\hat{s}_{i}\right) \hat{\omega}_{i, q}^{n+1} \\
\left(\hat{N}_{\text {trd }}^{+}[1]\right)_{i, q}^{n+1} & =\hat{\lambda}_{ \pm}\left(\hat{s}_{i}\right)\left[\sum_{k=0}^{L} \hat{\omega}_{i, q \mp k}^{n+1} \mathbb{P}\left[\vartheta_{L}^{ \pm}=k\right]+(\delta-\varepsilon) \mathbb{E}\left[\vartheta_{L}^{+}\right]\right] \\
\left(\hat{N}_{j m p}^{ \pm}[0]\right)_{i, q}^{n+1} & =\hat{h}_{ \pm}\left(\hat{s}_{i}\right)\left[\hat{\omega}_{0, \pm q}^{n+1}\right] \\
\left(\hat{N}_{j m p}^{ \pm}[1]\right)_{i, q}^{n+1} & =\hat{h}_{ \pm}\left(\hat{s}_{i}\right)\left[\hat{\omega}_{0, \pm q-L}^{n+1}+(-\delta-\varepsilon)\right]
\end{aligned}
$$

and the terminal and boundary conditions are given by:

$$
\begin{aligned}
\hat{\omega}_{i, q}^{N} & =-(\delta+\varepsilon)|q|, \\
\hat{\omega}_{M, q}^{n} & =\hat{\omega}_{M-1, q}^{n} .
\end{aligned}
$$

We rewrite the numerical scheme explicitely as:

$$
(\mathbb{I}-\Delta t A) \hat{\omega}^{n}=\hat{\omega}^{n+1}+\Delta t\left(Z^{n+1}+W\right)
$$

where:

$$
A=\operatorname{diag}(\hat{\kappa})+\frac{1}{\Delta \hat{s}} \operatorname{diag}(1, \ldots, 1,0) \operatorname{diag}(\hat{f}) \operatorname{tridiag}(0,-1,+1),
$$

with $A$ having size $M \times M, \operatorname{diag}(\hat{\kappa})$ and $\operatorname{diag}(\hat{f})$ being diagonal matrix with $\hat{\kappa}$ and $\hat{f}$ on the diagonal, and $\operatorname{tridiag}(0,1,-1)$ being the matrix with -1 on the diagonal and +1 on the 
upper diagonal. The multiplication by $\operatorname{diag}(1, \ldots, 1,0)$ allows to reproduce the Neumann condition given in (5.14). Furthermore:

$$
\begin{aligned}
W_{i, q}= & \hat{c}\left(\hat{s}_{i}\right) q-\bar{\eta} \hat{\sigma}^{2}\left(\hat{s}_{i}\right) q^{2} \\
Z_{i, q}^{n+1}= & \max _{\ell \in\{0,1\}:|q-\ell L| \in \mathbb{Y}}\left[\left(\hat{N}_{t r d}^{+}[l]\right)_{i, q}^{n+1}+\left(\hat{N}_{j m p}^{+}[l]\right)_{i, q}^{n+1}\right] \\
& +\max _{\ell \in\{0,1\}:|q+\ell L| \in \mathbb{Y}}\left[\left(\hat{N}_{t r d}^{-}[l]\right)_{i, q}^{n+1}+\left(\hat{N}_{j m p}^{-}[l]\right)_{i, q}^{n+1}\right]
\end{aligned}
$$

Proposition 5.1. The discretization scheme (5.15) is convergent.

Proof. In order to prove convergence of the numerical scheme, we need to prove monotonicity, stability and consistency. Since the numerical scheme is of Euler type, it is automatically consistent. Furthermore, the scheme is clearly monotone, since every negative multiplier of the value function is sent to the implicit part $A$. Finally, since the terminal condition $\hat{\omega}(T, \hat{s}, q)=-(\delta+\varepsilon)|q|$ is bounded on $\mathbb{Y}$, the monotonicity immediately implies stability, and we are done.

\subsection{Computational results}

We fix the following framework.

\begin{tabular}{cccc} 
parameter & symbol & value & \\
\hline horizon & $T$ & 5 minutes & \\
tick & $2 \delta$ & 1 \\
max order & $L$ & 1 \\
max inventory & $y_{\max }$ & 50 & \\
fees & $\varepsilon$ & 0.05 & \\
execution probability & $\vartheta_{L}^{+}=\vartheta_{L}^{-}=\vartheta$ & $\mathbb{P}[\vartheta=1]=0.05$ & (estimated) \\
risk adversion & $\eta$ & -0.33 & (estimated) \\
\hline adverse selection & $\rho$ & Figure 1 & (estimated) \\
jump intensity & $\hat{h}_{ \pm}$ & $\lambda_{0}=0.97, a=77.04, k=34.54$ &
\end{tabular}

\subsubsection{The value function}

An important factor affecting the shape of the value function and the optimal policy is given by the function

$$
\phi(t, p, i, s)=\mathbb{E}\left[P_{T} \mid I_{t}=i, S_{t}=s\right]
$$

corresponding to the expected gain at maturity obtained by holding one unit lot from $t$ to $T$. By the Feymann-Kac theorem, $\phi$ satisfies

$$
\begin{aligned}
\partial_{t} \phi+\partial_{s} \phi+h_{+}(s)[\phi(t, p+2 \delta i, i, 0)-\phi(t, p, i, s)] & \\
+h_{-}(s)[\phi(t, p-2 \delta i,-i, 0)-\phi(t, p, i, s)] & =0 \\
\phi(T, p, i, s) & =0 .
\end{aligned}
$$


By the ansatz $\phi(t, p, i, s)=p+i E(t, s)$, and again by the quantile change of variable $\hat{E}(t, \hat{s})=E(t, s)$, with $\hat{s}=\mathcal{Q} s$, we obtain that $\hat{E}$ satisfies:

$$
\begin{aligned}
\partial_{t} \hat{E}+\hat{f}(\hat{s}) \partial_{\hat{s}} \hat{E}+\hat{h}_{+}(\hat{s})[\hat{E}(0)-\hat{E}(\hat{s})+2 \delta]+\hat{h}_{-}(\hat{s})[-\hat{E}(0)-\hat{E}(\hat{s})-2 \delta] & =0 \\
\hat{E}(T, \hat{s}) & =0 .
\end{aligned}
$$

Figure 3 represents (the numerical solution for) $\hat{E}(t, \hat{s})$ and $q \hat{E}(t, \hat{s})$, showing how the change sign around the renewal median impacts the surface on the right, which is neither concave, nor convex. This is due to the fact that $\hat{h}_{+}(\hat{s})-\hat{h}_{-}(\hat{s})$ changes its sign too, as shown by Figure 1: we can easily prove by a comparison principle that if $\hat{h}^{-}>h^{+}$, then $\hat{E}<0$.

\section{$E^{\wedge}\left(t, \mathbf{s}^{\wedge}\right)$}

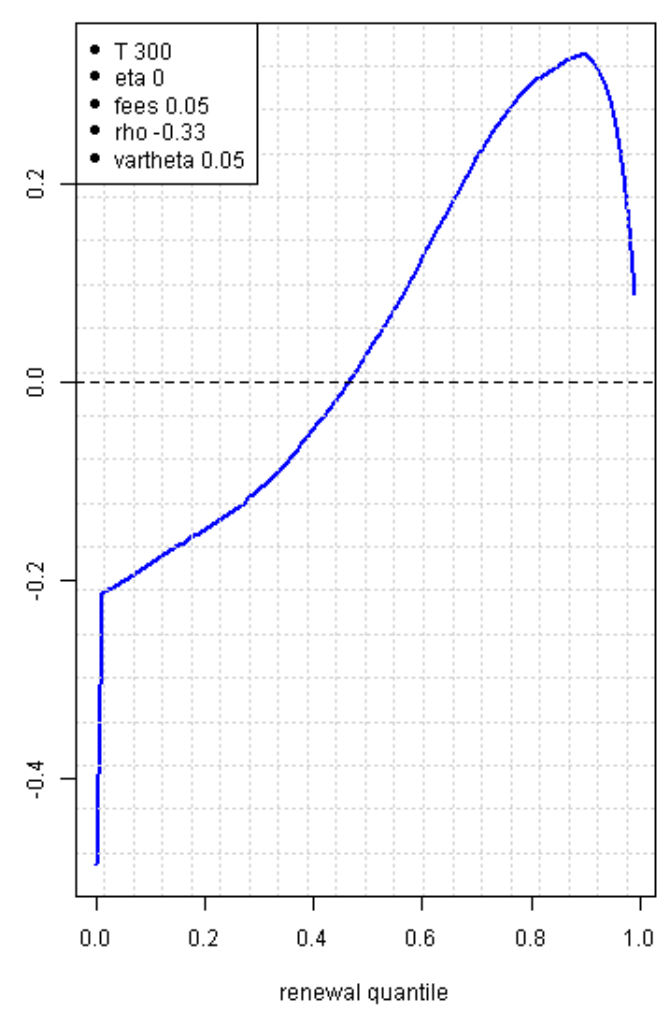

$q^{\star} E^{\wedge}\left(t, s^{\wedge}\right)$

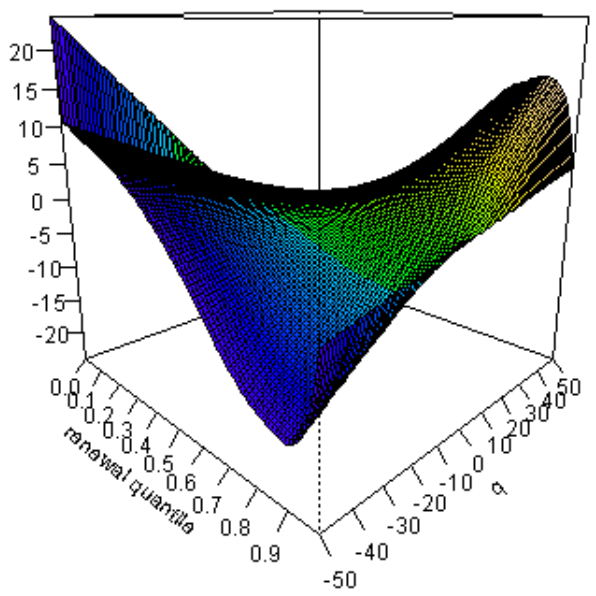

Figure 3: The conditional mean function function $\hat{E}(t, \hat{s})$

The main feature of the value function is the absence of concavity or convexity, as shown by Figures 4 and 5 . The value function is bigger for higher quantiles, mainly due to the fact that the price stabilizes, with $\hat{\sigma}^{2} \rightarrow 0$, leading to less risky market making, while the base trade intensity does not disappear $\left(\lim _{\hat{s} \rightarrow 1} \hat{\lambda}=\lambda_{0}>0\right)$. 


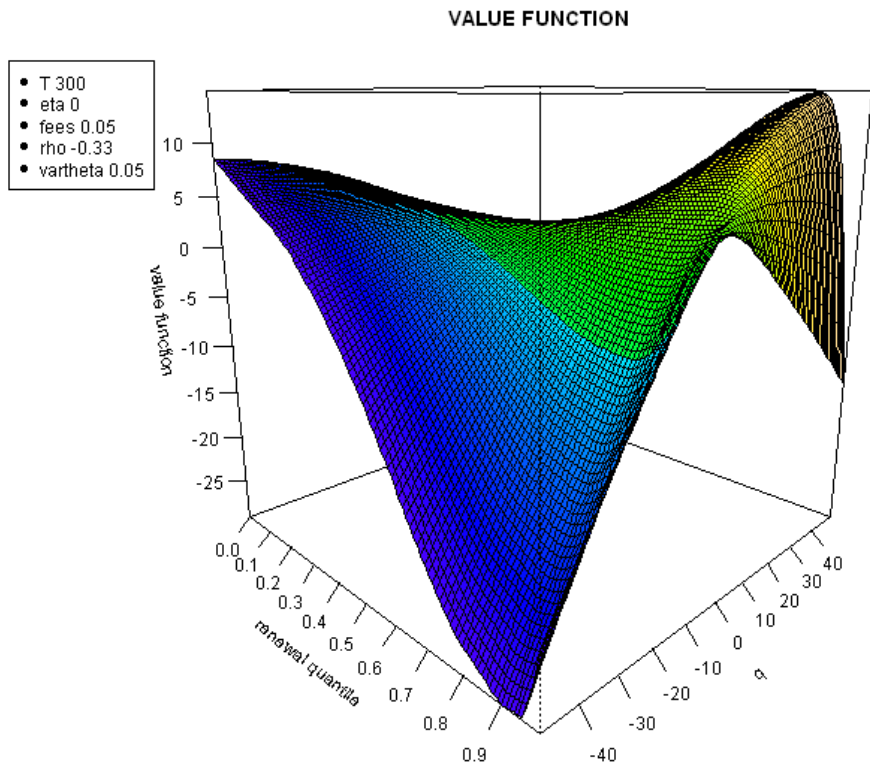

Figure 4: The value function $\hat{\omega}(0, \hat{s}, q)$

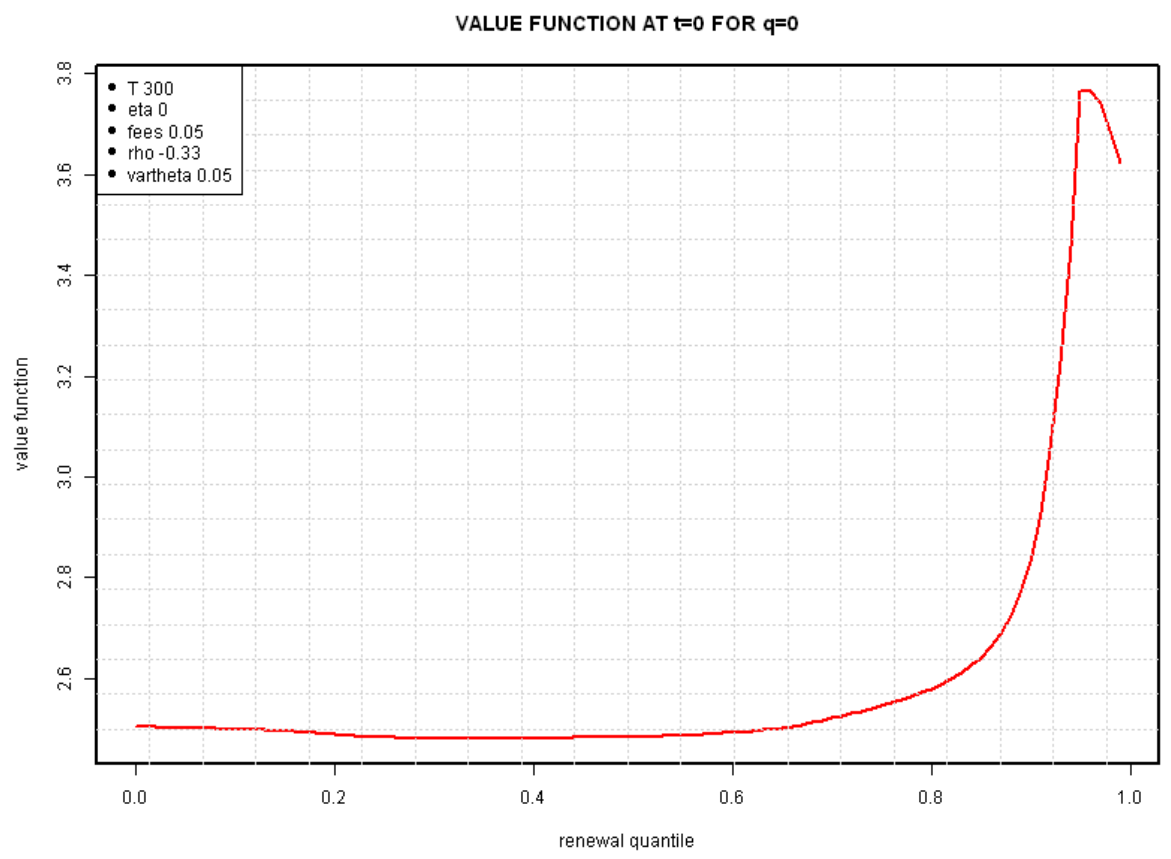

Figure 5: The value function $\hat{\omega}(0, \hat{s}, 0)$ 


\subsubsection{The optimal policy}

Figure 6 shows the optimal policy at time $t=0$. The + (resp. - ) region represents the zone where the agent should place a limit order on the concordant (resp. discordant) side: when $i=+$ this means the ask (resp. the bid) side, while if $i=-$ viceversa. One should keep in mind the $q$ is not the inventory, but the inventory multiplied by $i$, that we called strong inventory. In what follows we assume that $i=1$, so that $q=y,+=a s k$ and $-=b i d$. The optimal policy divides the plane into five well-distinguished regions that cross in two indifference points, where all the strategies are equivalent.

- The central region represents the no intervention zone, while on the topleft (resp. bottomleft) and the bottomright (resp. topright) the agent should be placed on one (resp. both) side (resp. sides).

- The particular symmetry around the $q=0$ axes is explained by the terminal condition $-|q|(\delta+\varepsilon)$, while the one around the antidiagonal takes its origin by particular shape of the function $\hat{E}(t, \hat{s})$, which changes sign around the renewal median.

- For $q>>0$ (resp. $q<<0$ ), the agent, who does not want to be exposed to the inventory risk, places her order so that to reduce her absolute inventory for most of the renewal quantiles.

- The agent does not play on one side only when the inventory risk and the gain given by holding a profitable position are in equilibrium. On the first diagonal the product $q \hat{E}(t, \hat{s})>0$ : the agent risk aversion leads her to play on the bid (resp. ask) side, while the expected gain due to the $\hat{E}$ leads her to play on the ask (resp. bid) one. Since the two forces balance each other, she decides to play on both sides (in order to gain the spread) or none, according to her absolute inventory, the trade intensity (higher for small renewal quantiles), the price drift (higher in absolute values for extreme renewal quantiles) and instability (higher for big renewal quantiles). 


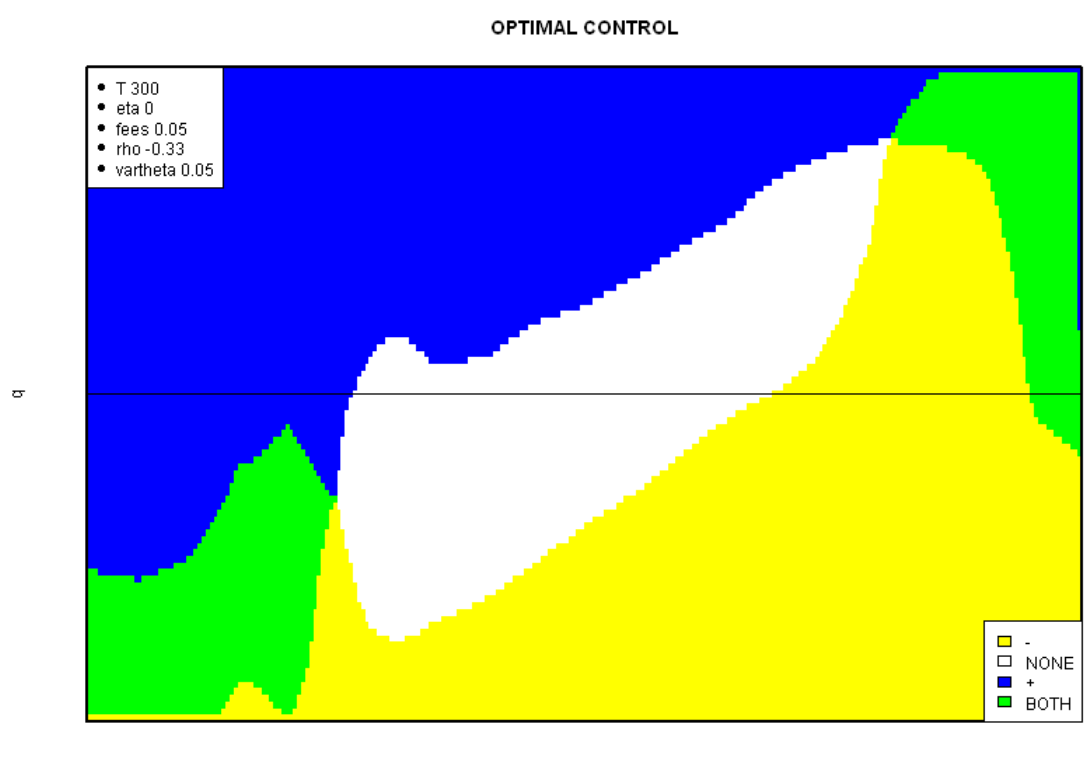

renewal quantile

Figure 6: The optimal control $\ell^{ \pm}(0, \hat{s}, q)$

\subsubsection{Impact of the parameter $\rho$ (adverse selection)}

By construction, the parameter $\rho$ contains information about the adverse selection risk of the agent. When $h_{-}(s)-h_{+}(s)>>0$, which is the case for small renewal quantiles where the trade intensity is strong, the price is likely to revert, and so the lower is $\rho$, the less the agent is likely to be executed on most favourable side, the strong one. Even though $h_{-}(s)-h_{+}(s)<0$ for high renewal quantiles, the impact of $\rho$ is smaller, since $h_{-}(s)-h_{+}(s)$, even if negative, is close to 0 , and trading intensity is weak. Figure 7 shows how the value function (all the other parameters fixed) is an increasing function of $\rho$ for small quantiles, while this phenomenon is inverted for $\hat{s} \approx 1$. This is reflected in the optimal policy displayed in Figure 8 by a smaller neutral zone (white) for $\rho=0$ than for negative $\rho$ in Figure 6 . 


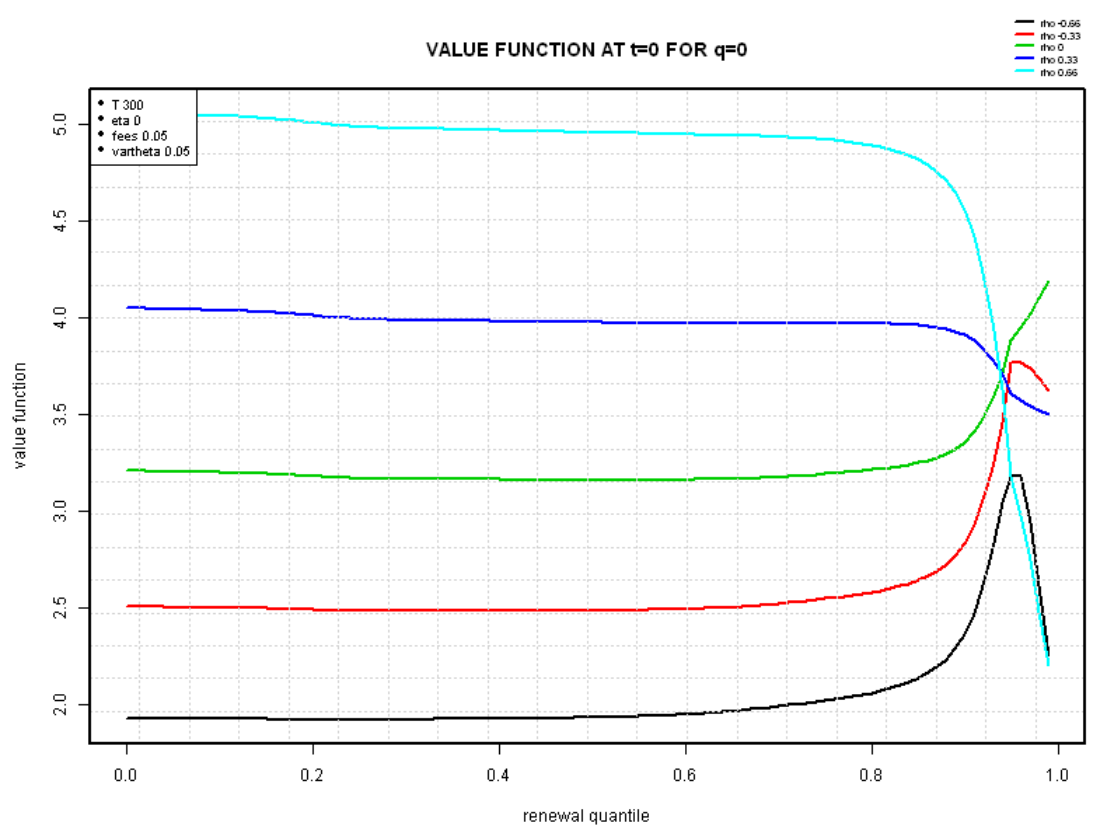

Figure 7: The value function $\hat{\omega}(0, \hat{s}, q)$ for different value of $\rho$

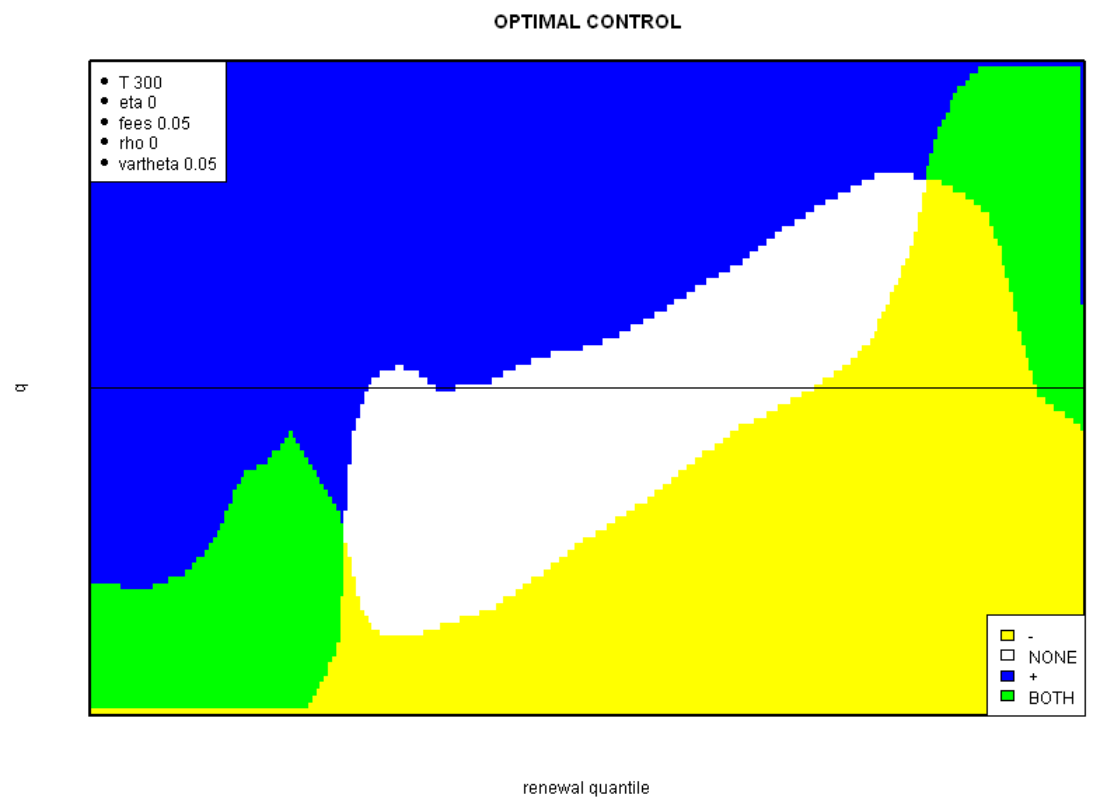

Figure 8: Optimal policy for $\rho=0$ and $\vartheta=0.05$, at $t=0$

\subsubsection{Impact of the parameter $\vartheta$ (execution probability)}

The parameter $\vartheta^{ \pm}$determines how often the agent is executed. As expected, the higher is the execution probability, the more the agent will gain, and the more she will be interested in playing, as shown in Figures 9 and 10. 


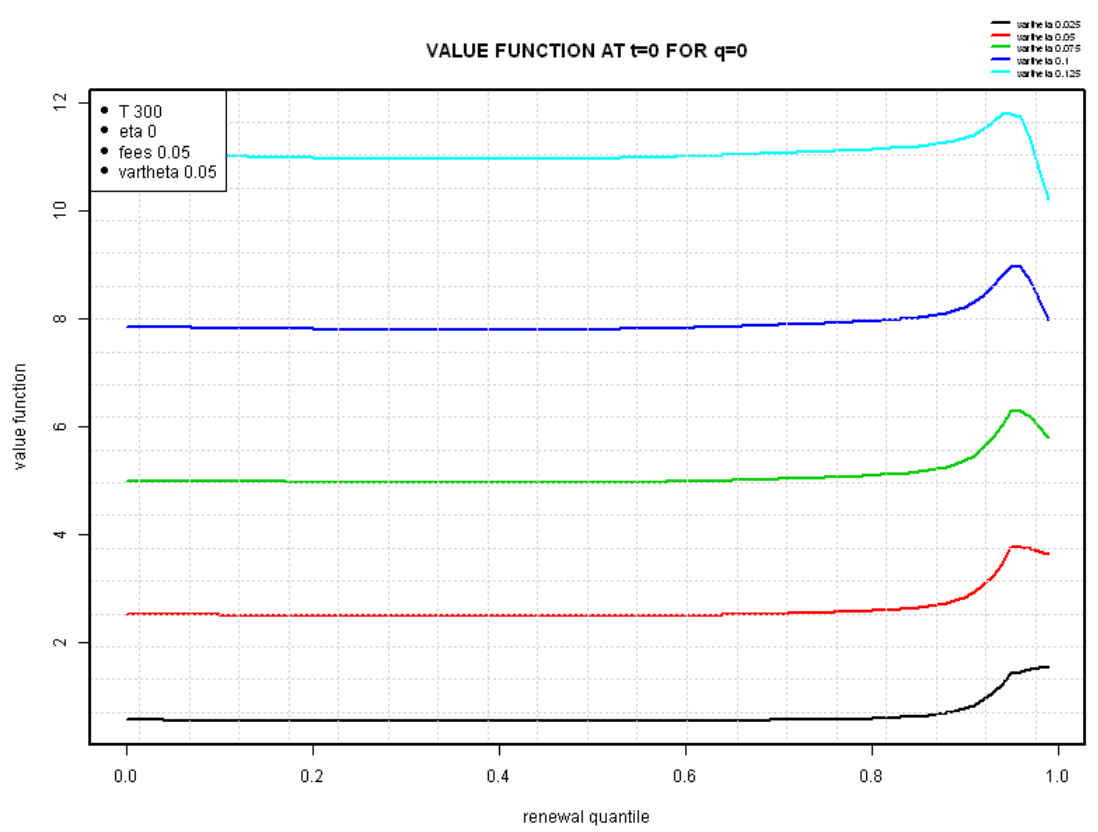

Figure 9: The value function $\hat{\omega}(0, \hat{s}, q)$ for different value of $\vartheta$

OPTIMAL CONTROL

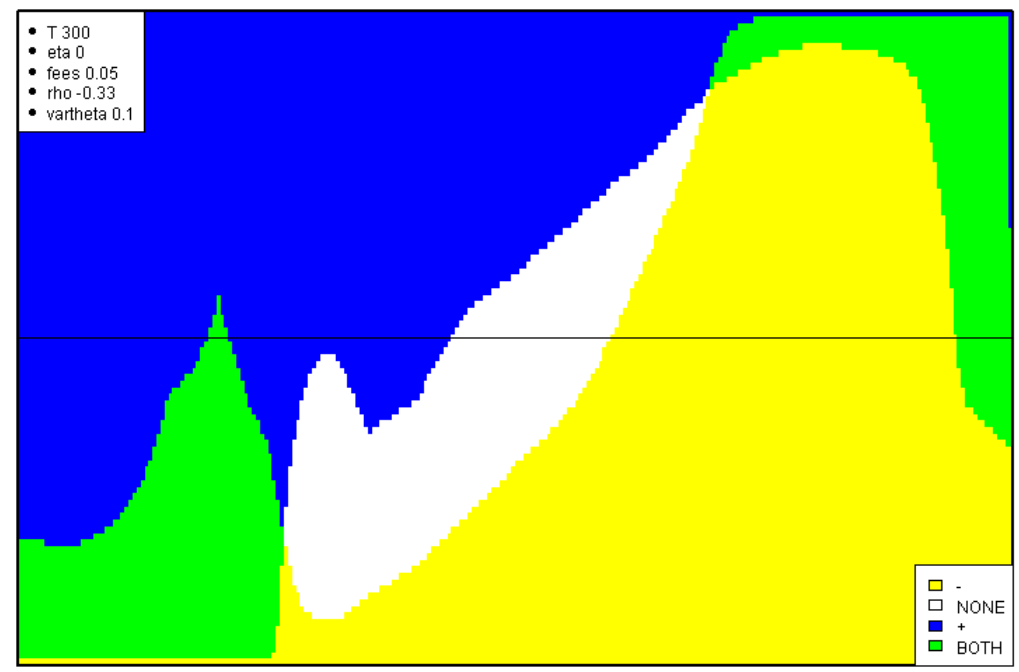

renewal quantile

Figure 10: Optimal policy for $\rho=0$ and $\vartheta=0.10$, at $t=0$ 


\section{References}

[1] F. Abergel and A. Jedidi. A mathematical approach to order book modeling. International Journal of Theoretical and Applied Finance (to appear), 2011.

[2] Y. Ait-Sahalia, P. Mykland, and L. Zhang. How often to sample a continuous-time process in the presence of market microstructure noise. The review of financial studies, 18:351-416, 2005.

[3] A. Alfonsi, A. Fruth, and A. Schied. Optimal execution strategies in limit order books with general shape functions. Quantitative Finance, 10:143-157, 2010.

[4] R. Almgren and N. Criss. Optimal execution of portfolio transactions. Journal of Risk, 3:5-39, 2000.

[5] M. Avellaneda and S. Stoikov. High frequency trading in a limit order book. Quantitative Finance, 8(3):217-224, 2008.

[6] L. Bauwens and N. Hautsch. Modelling financial high frequency data using point processes. Handbook of financial time series, Springer, 2nd edition, 2009.

[7] E. Bayraktar and M. Ludkovski. Liquidation in limit order books with controlled intensity. Mathematical Finance (to appear), 2011.

[8] R. Carmona and K. Webster. High frequency market making. 2012.

[9] A. Cartea and S. Jaimungal. Modeling asset prices for algorithmic and high frequency trading. Applied Mathematical Finance (to appear), 2011.

[10] R. Cont and A. de Larrard. Price dynamics in a markovian limit order market. SIAM Journal of Financial Mathematics, 4(1):1-25, 2010.

[11] P. Fodra and M. Labadie. High-frequency market-making with inventory constraints and directional bets. arXiv:1206.4810, 2012.

[12] P. Fodra and H. Pham. Semi markov model for market microstructure. arXiv:1305.0105, 2013.

[13] A. Gloter and J. Jacod. Diffusion with measurement errors. ESAIM: Proba. and Stats, 5:225$242,2001$.

[14] O. Guéant, C.A. Lehalle, and J.F. Tapia. Dealing with the inventory risk. a solution to the market making problem. Mathematics and financial economics, 7(4):477-507, 2013.

[15] F. Guilbaud and H. Pham. Optimal high-frequency trading with limit and market orders. Quantitative Finance, 13:79-94, 2013.

[16] X. Guo and M. Zervos. Optimal execution with multiplicative price impact. SIAM Journal of Financial Mathematics (to appear), 2013.

[17] M. Hoffmann, E. Bacry, S. Delattre, and J.F. Muzy. Modeling microstructure noise by mutually exciting point processes. Quantitative Finance, 13:65-77, 2013.

[18] B. Oksendal and A. Sulem. Applied Stochastic Control of Jump Diffusions. Springer, 2007.

[19] C.Y. Robert and M. Rosenbaum. A new approach for the dynamics of ultra high frequency data: the model with uncertainty zones. Journal of Financial Econometrics, 9(2):344-366, 2011. 